\title{
A Note on the Moment Generating Function for the Reciprocal Gamma Distribution
}

\section{By Staffan Wrigge}

\begin{abstract}
In this note we consider the function $\varphi(t)=\int_{0}^{\infty} e^{-t x} / \Gamma(x) d x$ and use the EulerMaclaurin expansion with the step-length $h=1 / 4$ to obtain some useful (from a numerical point of view) formulae. Numerical values of $\varphi(t)$ correct to $11 \mathrm{D}$ are given for $t=0.0(0.1) 5.0$.
\end{abstract}

Introduction. In [3] we analyzed the function

$$
\varphi(t)=\int_{0}^{\infty} \frac{e^{-t x}}{\Gamma(x)} d x
$$

and used the Euler-Maclaurin expansion to obtain some interesting (from a numerical point of view) formulae ((3.6) and (3.7)). These cases corresponded to the step-lengths $h=1$ and $h=\frac{1}{2}$. Using a little more sophisticated analysis, also the case $h=\frac{1}{4}$ may be investigated.

1. The Euler-Maclaurin Summation Formula With Step-Length $h=\frac{1}{4}$. We first must sum the expression

$$
F(t)=\sum_{k=0}^{\infty} \frac{e^{-k t / 4}}{\Gamma(k / 4)}
$$

It is evident that

$$
F(t)=\varphi_{1}(t)+\varphi_{2}(t)+\varphi_{3}(t)+\varphi_{4}(t)
$$

where

$$
\begin{aligned}
& \varphi_{1}(t)=\sum_{j=1}^{\infty} \frac{e^{-t j}}{\Gamma(j)}=e^{-t+e^{-t}} \\
& \varphi_{2}(t)=\sum_{j=0}^{\infty} \frac{e^{-t(j+1 / 2)}}{\Gamma(j+1 / 2)}=\frac{e^{-t / 2}}{\sqrt{\pi}}+\left(2 N\left(2^{1 / 2} e^{-t / 2}\right)-1\right) e^{-t+e^{-t}}, \\
& \varphi_{3}(t)=\sum_{j=0}^{\infty} \frac{e^{-(j+1 / 4) t}}{\Gamma(j+1 / 4)}, \\
& \varphi_{4}(t)=\sum_{j=0}^{\infty} \frac{e^{-(j+3 / 4) t}}{\Gamma(j+3 / 4)} .
\end{aligned}
$$

In the expression for $\varphi_{2}(t), N(\cdot)$ denotes the standardized normal distribution function, defined in [3, Eq. (3.5)].

Received September 21, 1982; revised June 22, 1983.

1980 Mathematics Subject Classification. Primary 33A15, 65D20; Secondary 44A10.

Key words and phrases. Reciprocal gamma distribution, generating function, Euler-Maclaurin formula. 
It remains to give useful alternative analytical expressions for the functions $\varphi_{3}(t)$ and $\varphi_{4}(t)$. We write $\varphi_{3}(t)$ in the form

$$
\varphi_{3}(t)=\frac{e^{-t / 4}}{\Gamma(1 / 4)}+\frac{e^{-t / 4}}{\Gamma(1 / 4)} \sum_{j=0}^{x} \frac{4^{j+1} e^{-(j+1) t}}{(4 j+1)(4 j-3) \cdots 1} .
$$

Putting $I=\int_{0}^{u} e^{-x^{4} / 4} d x$ and using integration by parts, we get

$$
I=\int_{0}^{u} e^{-x^{4} / 4} d x=\sum_{j=0}^{\infty} \frac{u^{4 j+1} e^{-u^{4} / 4}}{(4 j+1)(4 j-3) \cdots 1} .
$$

Thus we have

$$
e^{u^{4} / 4} \int_{0}^{u} e^{-x^{4} / 4} d x=\sum_{j=0}^{\infty} \frac{u^{4 j+1}}{(4 j+1)(4 j-3) \cdots 1} .
$$

Comparing (1.3) and (1.5), we get after some calculations

$$
\varphi_{3}(t)=\frac{1}{\Gamma(1 / 4)}\left\{e^{-t / 4}+e^{-t+e^{-t}}(\sqrt{2})^{3} \int_{0}^{\sqrt{2} e^{-t / 4}} e^{-x^{4} / 4} d x\right\} .
$$

In Section 2 we will discuss different techniques to evaluate numerically the integral occurring in (1.6).

Now write

$$
\varphi_{4}(t)=\frac{e^{-3 t / 4}}{\Gamma(3 / 4)}+\frac{e^{-3 t / 4}}{\Gamma(3 / 4)} \sum_{j=0}^{\infty} \frac{4^{j+1} e^{-(j+1) t}}{(4 j+3)(4 j-1) \cdots(3)} .
$$

Using integration by parts, we may prove that

$$
e^{u^{4} / 4} \int_{0}^{u} e^{-x^{4} / 4} x^{2} d x=\sum_{j=0}^{\infty} \frac{u^{4 j+3}}{(4 j+3)(4 j-1) \cdots(3)} .
$$

Comparing (1.7) and (1.8), we get

$$
\varphi_{4}(t)=\frac{1}{\Gamma(3 / 4)}\left\{e^{-3 t / 4}+\sqrt{2} e^{-t+e^{-t}} \int_{0}^{\sqrt{2} e^{-t / 4}} x^{2} e^{-x^{4} / 4} d x\right\} .
$$

We will return to the integral in (1.9) in Section 2.

Applying the Euler-Maclaurin summation formula to the function $\varphi(t)$ with a step-length $h=\frac{1}{4}$, we get, after some manipulations,

$$
\varphi(t)=\frac{1}{4} \sum_{i=1}^{4} \varphi_{i}(t)+\sum_{j=0}^{\infty} \frac{(-t)^{j}}{j !} \sum_{k=[(j+1) / 2]+1}^{\infty} \frac{B_{2 k}}{2 k}\left(\frac{1}{4}\right)^{2 k} a_{2 k-1-j} .
$$

(The coefficients $a_{n}$ occurring in (1.10) are defined in [3, Eq. (3.23)]). Using the methods developed in Section 2 for computing the functions $\varphi_{3}(t)$ and $\varphi_{4}(t)$, we tabulated $\varphi(t)$ to $15 \mathrm{D}$ in the interval $[0,5.0]$. See Table I, where we give only 12 decimals.

2. Calculation of Some Integrals. To calculate the functions $\varphi_{3}(t)$ and $\varphi_{4}(t)$ as given by (1.6) and (1.9) we need some fast and accurate methods to compute the integrals

$$
I=\int_{0}^{t} e^{-x^{4} / 4} d x \text { and } J=\int_{0}^{t} x^{2} e^{-x^{4} / 4} d x
$$


We start with $I$. Our first technique to evaluate this integral stems from a paper by Kerridge and Cook [2]. To generalize their arguments we must study the polynomials

$$
p_{n}(x)=(-1)^{n} e^{x^{4} / 4} D_{x}^{n} e^{-x^{4} / 4} .
$$

The polynomials $\left\{p_{n}(x)\right\}$ satisfy the recurrence relation

$$
\begin{aligned}
p_{n+1}(x)= & x^{3} p_{n}(x)-3 n x^{2} p_{n-1}(x)+3 x n(n-1) p_{n-2}(x) \\
& -n(n-1)(n-2) p_{n-3}(x),
\end{aligned}
$$

with starting values $p_{0}=1, p_{1}=x^{3}, p_{2}=x^{6}-3 x^{2}, p_{3}=x^{9}-9 x^{5}+6 x$. We now make the following Taylor series expansion

$$
I=\int_{0}^{t} e^{-x^{4} / 4} d x=\int_{0}^{t} \sum_{n=0}^{\infty}\left(x-\frac{t}{2}\right)^{n} \frac{\left(D_{x}^{n} e^{-x^{4} / 4}\right)_{x=t / 2}}{n !} d x .
$$

Carrying out the integration, we get

$$
I=t e^{-t^{4} / 64} \sum_{n=0}^{\infty} \frac{\left(\frac{1}{2} t\right)^{2 n}}{(2 n+1) !} p_{2 n}\left(\frac{1}{2} t\right)
$$

Define the polynomials $\theta_{n}(x)$ as

$$
\theta_{n}(x)=\frac{x^{n}}{n !} p_{n}(x)
$$

Then

$$
I=\int_{0}^{t} e^{-x^{4} / 4} d x=t e^{-t^{4} / 64} \sum_{n=0}^{\infty} \frac{\theta_{2 n}\left(\frac{1}{2} t\right)}{2 n+1},
$$

and the polynomials $\theta_{n}(x)$ satisfy the simpler recurrence relation

$$
\theta_{n+1}(x)=\frac{x^{4}}{n+1}\left(\theta_{n}(x)-3 \theta_{n-1}(x)+3 \theta_{n-2}(x)-\theta_{n-3}(x)\right),
$$

with starting values $\theta_{0}=1, \theta_{1}=x^{4}, \theta_{2}=\frac{1}{2}\left(x^{8}-3 x^{4}\right), \theta_{3}=\frac{1}{6}\left(x^{12}-9 x^{8}+6 x^{4}\right)$. To find the other technique, we study the expansion

$$
e^{-a^{2}(x-1 / 2)^{4} / 2}=e^{-a^{2} / 32} e^{a[a x(1-x)] / 4-[a x(1-x)]^{2} / 2} .
$$

Now remember that the Hermite polynomials may be defined by the following generating function (see, e.g., Kendall and Stuart [1, p. 155])

$$
e^{t z-t^{2} / 2}=\sum_{n=0}^{\infty} \frac{t^{n} H_{n}(z)}{n !} \text {. }
$$

Therefore

$$
e^{-a^{2}(x-1 / 2)^{4} / 2}=e^{-a^{2} / 32} \sum_{n=0}^{\infty} \frac{(x(1-x))^{n}}{n !} a^{n} H_{n}\left(\frac{a}{4}\right) .
$$

Integrating this identity between the limits 0 and 1 , we get after a few rearrangements

$$
\frac{1}{\sqrt{a}} \int_{-\sqrt{a} / 2}^{+\sqrt{a} / 2} e^{-u^{4} / 2} d u=e^{-a^{2} / 32} \sum_{n=0}^{\infty} \frac{a^{n} H_{n}(a / 4)}{n !(2 n+1)\left(\begin{array}{c}
2 n \\
n
\end{array}\right)}
$$


Making the proper manipulations, we finally get

$$
I=\int_{0}^{t} e^{-z^{4} / 4} d z=t e^{-t^{4} / 4} \sum_{n=0}^{\infty} \frac{2^{2 n}}{(2 n+1)\left(\begin{array}{c}
2 n \\
n
\end{array}\right)} \psi_{n}\left(\frac{t^{2}}{\sqrt{2}}\right),
$$

where $\psi_{n}(x)$ is defined by

$$
\psi_{n}(x)=\frac{x^{n}}{n !} H_{n}(x)
$$

$\psi_{n}(x)$ satisfies the recurrence relation

$$
\psi_{n+1}(x)=\frac{x^{2}}{n+1}\left(\psi_{n}(x)-\psi_{n-1}(x)\right) .
$$

If we instead consider the expression $a\left(x-\frac{1}{2}\right)^{2} e^{-a^{2}(x-1 / 2)^{4} / 2}$ and proceed along the lines indicated by (2.9)-(2.12), we get

$$
\begin{aligned}
J= & \int_{0}^{t} z^{2} e^{-z^{4} / 4} d z=t^{3} e^{-t^{4} / 4} \sum_{n=0}^{\infty} \frac{\psi_{n}\left(t^{2} / \sqrt{2}\right) 2^{2 n}}{(2 n+1)\left(\begin{array}{c}
2 n \\
n
\end{array}\right)} \\
& -4 t^{3} e^{-t^{4} / 4} \sum_{n=0}^{\infty} \frac{\psi_{n}\left(t^{2} / \sqrt{2}\right) 2^{2 n}}{(2 n+3)\left(\begin{array}{c}
2 n+2 \\
n+1
\end{array}\right)} .
\end{aligned}
$$

Some simplifications finally yield

$$
J=\int_{0}^{t} z^{2} e^{-z^{4} / 4} d z=t^{3} e^{-t^{4} / 4} \sum_{n=0}^{\infty} \frac{\psi_{n}\left(t^{2} / \sqrt{2}\right) 2^{2 n}}{(2 n+3)(2 n+1)\left(\begin{array}{c}
2 n \\
n
\end{array}\right)} .
$$

To obtain a formula for $J$ similar to (2.7) we must introduce the polynomials

$$
P_{n}(x)=(-1)^{n} e^{x^{4} / 4} D_{x}^{n} x^{2} e^{-x^{4} / 4} .
$$

In terms of the polynomials $p_{n}(x)$ we may write

$$
P_{n}(x)=x^{2} p_{n}(x)-2 n x p_{n-1}(x)+n(n-1) p_{n-2}(x) .
$$

If we define the functions $\left\{\xi_{n}(x)\right\}$ as

$$
\xi_{n}(x)=x^{n} P_{n}(x) / n !
$$

we observe that

$$
\xi_{n}(x)=x^{2}\left(\theta_{n}(x)-2 \theta_{n-1}(x)+\theta_{n-2}(x)\right)=x^{2} \Delta^{2} \theta_{n}(x),
$$

and we get

$$
J=\int_{0}^{t} x^{2} e^{-x^{4} / 4} d x=t e^{-t^{4} / 64} \sum_{n=0}^{\infty} \frac{\xi_{2 n}\left(\frac{1}{2} t\right)}{2 n+1} .
$$

It is evident that for small values of $t$ we may use the simple formula

$$
\int_{0}^{t} x^{a} e^{-x^{4} / 4} d x=\sum_{n=0}^{\infty} \frac{(-1)^{n} t^{4 n+a+1}}{n ! 4^{n}(4 n+a+1)} ; \quad a \in\{0,2\} .
$$

When calculating the integrals $I$ and $J$ we found formulae (2.7) and (2.22) to be of the greatest value. The convergence in the series (2.13) and (2.17) turned out to be rather slow. For small values of $t$ also the formula (2.23) was useful. The resulting numerical values of $\varphi(t)$ correct to (at least) 11D appear in Table I. The reason why 
we give 12D in Table I is that a use of Watson's Lemma [3, Eq. (3.23)] indicates that for $t=5.0$ we have a precision of 14D. A comparison with Table IV of [3] confirms that Table IV correctly yields 10D.

\section{TABLE I}

Values of $\varphi(t)$ using the polynomials $p_{n}(x)$.

$\begin{array}{cccccc}t & \varphi(t) & t & \varphi(t) & t & \varphi(t) \\ 0.0 & 2.807770242028 & 1.7 & 0.300933511958 & 3.4 & 0.091421032961 \\ 0.1 & 2.326237047400 & 1.8 & 0.275394801591 & 3.5 & 0.086581618381 \\ 0.2 & 1.946771821817 & 1.9 & 0.252780695525 & 3.6 & 0.082102419937 \\ 0.3 & 1.644358498906 & 2.0 & 0.232680797724 & 3.7 & 0.077949589282 \\ 0.4 & 1.400823696157 & 2.1 & 0.214751780293 & 3.8 & 0.074093068791 \\ 0.5 & 1.202793433329 & 2.2 & 0.198705012619 & 3.9 & 0.070506103326 \\ 0.6 & 1.040305961681 & 2.3 & 0.184296711962 & 4.0 & 0.067164822585 \\ 0.7 & 0.905856615825 & 2.4 & 0.171320056208 & 4.1 & 0.064047882795 \\ 0.8 & 0.793731332327 & 2.5 & 0.159598832949 & 4.2 & 0.061136158425 \\ 0.9 & 0.699535729986 & 2.6 & 0.148982298581 & 4.3 & 0.058412476217 \\ 1.0 & 0.619858414145 & 2.7 & 0.139340995782 & 4.4 & 0.055861385110 \\ 1.1 & 0.552027547158 & 2.8 & 0.130563334193 & 4.5 & 0.053468956707 \\ 1.2 & 0.493932984351 & 2.9 & 0.122552782029 & 4.6 & 0.051222611791 \\ 1.3 & 0.443895013087 & 3.0 & 0.115225549144 & 4.7 & 0.049110969132 \\ 1.4 & 0.400566564690 & 3.1 & 0.108508667380 & 4.8 & 0.047123713408 \\ 1.5 & 0.362859707863 & 3.2 & 0.102338393501 & 4.9 & 0.045251479573 \\ 1.6 & 0.329889922708 & 3.3 & 0.096658875253 & 5.0 & 0.043485751382\end{array}$

National Defence Research Institute

Division I, Section 123

S-102 54 Stockholm, Sweden

1. M. G. Kendall \& A. Stuart, The Aduanced Theory of Statistics, Vol. I, Charles Griffin \& Company Limited, 1958.

2. D. F. Kerridge \& G. W. Cook, "Yet another series for the normal integral," Biometrika, v. 63, 1976, pp. 401-403.

3. A. FransÉn \& S. WRIGGE, "Calculation of the moments and the moment generating function for the reciprocal gamma distribution," Math. Comp., v. 42, 1984, pp. 601-616. 